\title{
The Influencing Factors of Nurse image and Ego-Resilience on Nursing Professional Values of Nursing Students
}

\author{
Gyung Park1)
}

\begin{abstract}
This study was a descriptive research to examine the factors influencing Nursing professional values of nursing students. This study was performed with structured questionnaire to undergraduates in nursing departments at one university in one City from September 23 to 27, 2019. The subjects were 193 nursing students majoring in nursing in one university located in J Province. Data were analyzed using SPSS/WIN 22.0, averages, standard deviations, t-tests, ANOVA, Schéffe's test, Pearson's correlation coefficients and multiple regressions. As a result, Nurse image by general characteristics showed a significant differences in personality, thinking for nursing professionalism. Ego-resilience showed a significant difference in age, sex, grade, personality, thinking for nursing professionalism, and application motivation. Nursing professional values showed a significant difference in grade, personality, and thinking for nursing professionalism. An analysis of the correlations between Nurse image, Ego-resilience and nursing professional values indicated the existence of a significant differences correlation between nurse image and Ego-resilience. The factors influencing on Nursing professional values indicated that Nurse image, Ego-resilience. Regression model was showed statistically significant, and the model's explanatory power was $56.7 \%$. Therefore, Nursing professional values of nursing students can be enhanced if theoretical curricula and extra-curricular programs to improve Nurse image and Ego-resilience are developed and applied appropriately.
\end{abstract}

Keywords: Nurse Image, Ego-resilience, Nursing Professional Values, Nursing College Students

\section{Introduction}

\subsection{Necessity of this Research}

With the increased demand for nurses due to changes in medical environments, the government of Korean gradually increased the enrollment quota of nursing colleges by 8,000 over the past decade (2008 to 2018). However, hospitals still suffer from shortage of nurses. Although nearly 20,000 students graduate from nursing colleges every year, the number of nurses working at medical institutions per 1,000 people in Korea is $53.8 \%$ of the average offered by the Organization for Economic Cooperation and Development (OECD). Out of the total 375,000

Received(May 07, 2020), Review Result(1st: June 24, 2020, 2nd: August 12, 2020), Accepted(September 25, 2020)

1) (Assistant Professor) 58607 Dept. Nurse Science, Mokpo Catholic University, Youngsanro 697, Mokpo city, Jellanamdo, South Korea

email: gypark@mcu.ac.kr 
license holders as nurses, 186,000 are currently working at medical institutions, accounting for only $49.6 \%$ of the holders (as of 2017). This shortage of nurses who are working at medical institutions may be related to the report of the Hospital Nurses Association, according to which the within-one-year turnover rate of nurses who were employed in hospitals after graduating nursing colleges merely reaches $33.9 \%[1]$.

Nursing professional values are closely associated with job satisfaction, job performance, and organizational commitment of nurses. Nurses with more positive nursing professional values are reported to show higher job satisfaction, lower turnover rate, more effective performance[2] and higher organizational commitment[3]. Given that nursing professional values formed in colleges tend to be led to those of clinical nurses[4]. Education in nursing colleges serves as a basis for establishing and solidifying the values of nurses, playing an important role in improving and developing the quality of nursing service[5]. Nursing students who established desirable nursing professional values through nursing education can further develop their values after becoming nurses. Therefore, it is required to pay attention to establishing and educating the nursing professional values of nursing students[6].

Nurse image of nursing students allows the students to change their views on nursing and to accept knowledge, skills, attitudes, beliefs, and ethical standards of nursing as an internalized part of their behaviors during nursing education. Positive images on nurses enhance the self-esteem of nursing students, playing a significant role in establishing their identity as nurses[7]. Nursing students who have a positive image of nurses during nursing education have a positive influence on performing their roles in clinical practice[8]. In addition, positive images of nurses are reported as the most influential variable in establishing nursing professional values[9]. Considering the effects of an image of a professional job on professional values, nursing professional values, along with nurse image, should be one of the variables to be considered[10].

Ego-resilience is an ability to adjust self-control levels to respond to stressful situations[11] flexibly. Considering the effects of an image of a professional job on professional values, nursing professional values, along with nurse image, should be one of the variables to be considered[10]. This is why ego-resilience is an essential internal factor for nursing students who will work in various clinical sites[12], the result of precedent studies showed that nursing students with higher ego-resilience have higher nursing professional values[13]. As such, the Korean Accreditation Board of Nursing Education emphasizes nursing professional values as a concept of measuring major educational outcomes that nursing students should achieve through college curricula[14]. 
Precedent studies have reported a variety of variables that affect the nursing professional values of nursing students, including nurse image[15][16], ego-resilience[17][18], professional self-concept[15], critical thinking[18], satisfaction with major[19], self-leadership[19], and empowerment[14]. However, few studies have been performed on the correlations between nurse image, ego-resilience, and nursing professional values. The purpose of this study is to identify these correlations, determine factors affecting nursing professional values, and provide a basic data in order to prepare educational interventions for establishing nursing professional values of nursing students.

\subsection{Research Objectives}

The purpose of this study is to identify the correlations between Nurse image, Ego-resilience, and Nursing professional values of nursing students and to determine the factors affecting their Nursing professional values. The concrete objectives are as follows:

(1) To identify general characteristics of the subjects.

(2) To identify Nurse image, Ego-resilience, and Nursing professional values of the subjects.

(3) To determine differences in Nurse image, Ego-resilience, and Nursing professional values of the subjects based on their general characteristics.

(4) To determine the correlations between Nurse image, Ego-resilience, and Nursing professional values of the subjects.

(5) To identify the factors influencing Nursing professional values of the subjects.

\section{Research Methods}

\subsection{Research Design}

This study is a descriptive survey study to determine the correlations between Nurse image, Ego-resilience, and Nursing professional values of nursing students and to determine the factors affecting their Nursing professional values.

\subsection{Subjects and Data Collection}

The subjects of this study were students of a nursing college who understood the purpose of this study and agreed to participate. Although the number of subjects that is necessary to maintain .20 of the effect size, .05 of the significance level, and .85 of the power was calculated 
as 182 by the $G$ * Power 3.1, this study distributed 200 structured questionnaires considering the dropout rate. Seven of them were excluded due to unsatisfactory responses, and a total of 193 questionnaires were used for the final analysis. The questionnaires were distributed between September 23 and 27, 2019, requiring approximately 20 minutes to complete. Before the subjects completed the questionnaires, the researchers explained the purpose and content of the study, specifying that collected data would be processed as anonymous, would not be used for any purpose other than the research, and would be withdrawn at any time upon the subject's request.

\subsection{Research Tools}

\subsubsection{Nurse Image}

To measure Nurse image, a tool by Kim[20] was used by revising and supplementing the tool of Lee[21] and Yang[22] based on the tool presented by Song[23] for measuring the images of nurses from physicians, nurses, hospital employees, and general public. With a total of 40 items, Kim's tool adopted the 5-point Likert scale: "Not at all" is 1 point and "Very much" is 5 points. The higher the score, the more positive the Nurse image. In the study of Kim[23], the reliability of the tool was revealed as Cronbach's $a=.95$ and in this study, Cronbach's a was 92 .

\subsubsection{Ego-resilience}

To measure Ego-resilience, a tool by You and Shim[24] was used by translating and revising the Ego-resilience tool of Block and Kremen[11]. The tool adopted a 4-point scale and consisted of a total of 14 items, which are divided into three items of interpersonal relationship, five of curiosity, two of emotional control, two of vitality, and two of optimism. The scores of the items were ranged from 1 point ("Not at all") to 4 points ("Very much"). The higher the score, the higher the Ego-resilience. The Cronbach's a in the study of Block and Kremen[11] was .76 and in this study, Cronbach's a was .82 .

\subsubsection{Nursing Professional Values}

To measure Nursing professional values, the 18 items that were reduced from the 29 items (developed by Yeun et al.[25]) by Han et al.[2] through verifying construct validity via factor analysis were used. The tool was divided into five sub-domains of professional self-concept (six items), social perception (five items), nursing professionalism (three items), roles of nursing (two 
items), and autonomy of nursing (two items). It adopted the 5-point Likert scale: "Not at all" is 1 point and "Very much" is 5 points. As the negative items were counted backward, the higher the total score, the more positive the Nursing professional values. The reliability of the tool in the study of Han et al.[2] was revealed as Cronbach's $a=.91$ and in this study, Cronbach's a was .86 .

\subsection{Data Analysis}

The SPSS/WIN 22.0 program analyzed the collected data.

- The general characteristics of the subjects were determined by number and percentage, while Nurse image, Ego-resilience, and Nursing professional values of the subjects were determined by mean and standard deviation.

- The differences in Nurse image, Ego-resilience, and Nursing professional values of the subjects based on their general characteristics were analyzed with t-test and ANOVA, while Schéffe's test was used as post hoc test.

- Pearson's correlation coefficients were used to determine the correlations between Nurse image, Ego-resilience, and Nursing professional values of the subjects.

- Multiple regression was used to identify the factors affecting Nursing professional values of the subjects.

\section{Results}

\subsection{General Characteristics of Subjects}

Most of the subjects were aged between 20 to 24 years old 170 (88.1\%). There were 165 females(85.5\%) and 28 males(14.5\%). Juniors were $89(46.1 \%)$ while seniors were 104 (53.9\%). Among the subjects, 96 (49.7\%) were religious while 97(50.3\%) answered they had no religion. As for personality, $17(8.8 \%)$ said they were "Very optimistic," 141 (73.1\%) "Optimistic," 33 (17.1\%) "Pessimistic," and 2 (1.0\%) "Very pessimistic." While 162 (84.0\%) were optimistic about nursing professionalism, $31(16.0 \%)$ were pessimistic. The motivations of selecting nursing were "Employment guarantee" 95 ( 49.2\%), "Aptitude" 38 (19.7\%), "High school record" 10 (5.2\%), "Recommendation of parent or others" 43 (22.3\%), and "Service to others" 7 (3.6\%) (see [Table 1]). 
[Table 1] General Characteristics of Subjects

$(\mathrm{N}=193)$

\begin{tabular}{|c|c|c|c|}
\hline Characteristics & Categories & $\mathrm{n}$ & $\%$ \\
\hline \multirow{3}{*}{ Age } & $20-24$ & 170 & 88.1 \\
\hline & $25-29$ & 19 & 9.8 \\
\hline & $30-34$ & 4 & 2.1 \\
\hline \multirow{2}{*}{ Sex } & Female & 165 & 85.5 \\
\hline & Male & 28 & 14.5 \\
\hline \multirow{2}{*}{ Grade } & Junior & 89 & 46.1 \\
\hline & Senior & 104 & 53.9 \\
\hline \multirow{2}{*}{ Religion } & Yes & 96 & 49.7 \\
\hline & No & 97 & 50.3 \\
\hline \multirow{4}{*}{ Personality } & Very optimistic & 17 & 8.8 \\
\hline & Optimistic & 141 & 73.1 \\
\hline & Pessimistic & 33 & 17.1 \\
\hline & Very pessimistic & 2 & 1.0 \\
\hline \multirow{2}{*}{$\begin{array}{l}\text { Thinking for nursing } \\
\text { professionalism }\end{array}$} & Optimistic & 162 & 84.0 \\
\hline & Pessimistic & 31 & 16.0 \\
\hline \multirow{5}{*}{ Application motivation } & Employment guarantee & 95 & 49.2 \\
\hline & Aptitude & 38 & 19.7 \\
\hline & High school record & 10 & 5.2 \\
\hline & Recommendation of parent or others & 43 & 22.3 \\
\hline & service to others & 7 & 3.6 \\
\hline
\end{tabular}

\subsection{Level of Ego-resilience, Nurse Image and Nursing Professional Values of Subjects}

The Nurse image of the subjects averaged 3.87 $( \pm 0.51)$, the Ego-resilience $3.37( \pm 0.52)$, and the Nursing professional values $3.68( \pm 0.47)$ out of 5 points(see [Table 2]).

[Table 2] Mean Score of Nurse Image, Ego-resilience, and Nursing Professional Values

$(\mathrm{N}=193)$

\begin{tabular}{l|l|l|l}
\hline Variables & Mean \pm SD & Min & Max \\
\hline \hline Nurse image & $3.87 \pm 0.51$ & 2.35 & 4.93 \\
Ego-resilience & $3.37 \pm 0.52$ & 2.25 & 4.85 \\
Nursing professional values & $3.68 \pm 0.47$ & 2.22 & 4.89 \\
\hline
\end{tabular}

\subsection{Differences in Nurse Image, Ego-resilience and Nursing Professional Values of Subjects}

The Nurse image based on the general characteristics of the subjects showed a significant difference in personality $(\mathrm{F}=7.601, p<.001)$ and thinking for nursing professionalism $(\mathrm{t}=4.874, p$ $<.001)$. The Ego-resilience showed a significant difference in age $(\mathrm{F}=2.605, p=.043)$, sex $(\mathrm{t}=-2.420$, $\left.p^{F} .015\right)$, grade $(\mathrm{t}=3.683, p=.034)$, personality $(\mathrm{F}=21.651, p<.001)$, thinking for nursing 
professionalism $(\mathrm{t}=2.563, p=.013)$, and application motivation $(\mathrm{F}=2.542, p=.034)$. Nursing professional values have significant difference in grade $(\mathrm{F}=3.374, p=036)$, personality $(\mathrm{F}=6.218, p$ $<.001)$, and thinking for nursing professionalism $(\mathrm{t}=5.993, p<.001)$ (see [Table 3]).

The results of Posteriori analysis showed that the students with "Very optimistic" personality had higher scores of Nurse image than those with "Pessimistic" and "Very pessimistic" personality. As for the Ego-resilience based on personality, "Very optimistic" students showed higher scores than "Optimistic," "Pessimistic," or "Very pessimistic" ones, and the "Optimistic" students showed higher scores than "Pessimistic" ones. When application motivations were considered, "Aptitude" induced higher scores than "Recommendation of parent or others" or "Employment guarantee." As for the Nursing professional values based on personality, "Very optimistic" students showed higher scores than "Pessimistic" or "Very pessimistic" ones.

[Table 3] Differences in Nurse image, Ego-resilience, and Nursing professional values $(\mathrm{N}=193)$

\begin{tabular}{|c|c|c|c|c|c|c|c|c|c|c|}
\hline \multirow{2}{*}{ Characteristics } & \multirow{2}{*}{ Categories } & \multicolumn{3}{|c|}{ Nurse image } & \multicolumn{2}{|c|}{ Ego-resilience } & \multicolumn{4}{|c|}{$\begin{array}{l}\text { Nursing } \\
\text { professional values }\end{array}$} \\
\hline & & $\mathrm{M} \pm \mathrm{SD}$ & $\begin{array}{l}t \text { or F } \\
\text { (Scheffe) }\end{array}$ & $p$ & $\mathrm{M} \pm \mathrm{SD}$ & $\begin{array}{c}t \text { or F } \\
\text { (Scheffe) }\end{array}$ & $p$ & $\mathrm{M} \pm \mathrm{SD}$ & $\begin{array}{c}t \text { or F } \\
\text { (Scheffe) }\end{array}$ & $p$ \\
\hline \multirow{3}{*}{ Age } & $20-24 a$ & $3.86 \pm 0.51$ & & & $3.35 \pm 0.53$ & \multirow{3}{*}{2.605} & \multirow{3}{*}{.043} & $3.68 \pm 0.46$ & \multirow{3}{*}{0.835} & \multirow{3}{*}{.476} \\
\hline & $25-29 b$ & $3.95 \pm 0.45$ & 0.741 & .442 & $3.92 \pm 0.61$ & & & $3.79 \pm 0.50$ & & \\
\hline & $30-34 c$ & $3.84 \pm 0.32$ & & & $3.31 \pm 0.33$ & & & $3.43 \pm 0.24$ & & \\
\hline \multirow{2}{*}{ Sex } & Female & $3.82 \pm 0.43$ & \multirow{2}{*}{-0.243} & \multirow{2}{*}{.801} & $3.33 \pm 0.45$ & \multirow{2}{*}{-2.420} & \multirow{2}{*}{.015} & $3.68 \pm 0.46$ & \multirow{2}{*}{-.340} & \multirow{2}{*}{.734} \\
\hline & Men & $3.85 \pm 0.50$ & & & $3.60 \pm 0.53$ & & & $3.70 \pm 0.49$ & & \\
\hline \multirow{2}{*}{ Grade } & Junior & $3.81 \pm 0.46$ & \multirow{2}{*}{1.452} & \multirow{2}{*}{.231} & $3.33 \pm 0.43$ & \multirow{2}{*}{3.683} & \multirow{2}{*}{.034} & $3.64 \pm 0.46$ & \multirow{2}{*}{3.374} & \multirow{2}{*}{.036} \\
\hline & Senior & $3.82 \pm 0.44$ & & & $3.43 \pm 0.52$ & & & $3.62 \pm 0.48$ & & \\
\hline \multirow[t]{2}{*}{ Religion } & Yes & $3.86 \pm 0.47$ & \multirow[t]{2}{*}{0.331} & \multirow[t]{2}{*}{.664} & $\begin{array}{l}3.234 \pm 0.5 \\
3\end{array}$ & \multirow[t]{2}{*}{1.623} & \multirow[t]{2}{*}{.142} & $3.69 \pm 0.48$ & \multirow[t]{2}{*}{0.351} & \multirow[t]{2}{*}{.726} \\
\hline & No & $3.85 \pm 0.45$ & & & $3.44 \pm 0.45$ & & & $3.67 \pm 0.46$ & & \\
\hline \multirow{4}{*}{ Personality } & $\begin{array}{l}\text { Very } \\
\text { optimistic }\end{array}$ & $4.11 \pm 0.50$ & \multirow{4}{*}{$\begin{array}{l}7.601 \\
(a>c, d)\end{array}$} & \multirow{4}{*}{$<001$} & $3.85 \pm 0.54$ & & & $3.93 \pm 0.44$ & & \\
\hline & Optimisticb & $3.86 \pm 0.43$ & & & $3.33 \pm 0.52$ & 21.651 & $<001$ & $3.69 \pm 0.43$ & 6.218 & $<00$ \\
\hline & Pessimisticc & $3.70 \pm 0.52$ & & & $3.12 \pm 0.42$ & $(a>b, c, d)$, & & $3.55 \pm 0.53$ & $(a>c, d)$ & $<$ < \\
\hline & $\begin{array}{l}\text { Very } \\
\text { pessimisticd }\end{array}$ & $3.16 \pm 0.85$ & & & $3.10 \pm 0.61$ & & & $2.98 \pm 0.98$ & & \\
\hline Thinking for & Optimistic & $3.95 \pm 0.48$ & 4874 & & $3.33 \pm 0.52$ & & & $3.75 \pm 0.44$ & & \\
\hline $\begin{array}{l}\text { nursing } \\
\text { professionalism }\end{array}$ & Pessimistic & $3.54 \pm 0.53$ & $4.0 / 4$ & $<001$ & $3.24 \pm 0.45$ & 2.563 & 013 & $3.30 \pm 0.46$ & 5.993 & $<001$ \\
\hline & $\begin{array}{l}\text { Employment } \\
\text { a guarantee }\end{array}$ & $3.86 \pm 0.45$ & & & $3.43 \pm 0.52$ & & & $3.67 \pm 0.49$ & & \\
\hline $\begin{array}{l}\text { Application } \\
\text { motivation }\end{array}$ & Aptitudeb & $3.95 \pm 0.43$ & 1.083 & .342 & $3.54 \pm 0.53$ & $\begin{array}{l}2.542 \\
(b>a, d)\end{array}$ & .034 & $3.81 \pm 0.45$ & 1.685 & .154 \\
\hline & $\begin{array}{l}\text { High school } \\
\text { recordc }\end{array}$ & $3.85 \pm 0.42$ & & & $3.43 \pm 0.42$ & & & $3.58 \pm 0.56$ & & \\
\hline
\end{tabular}




\begin{tabular}{|c|c|c|c|}
\hline $\begin{array}{l}\text { Recommend } \\
\text { ation of } \\
\text { parent or } \\
\text { othersd }\end{array}$ & $3.76 \pm 0.56$ & $3.42 \pm 0.50$ & $3.61 \pm 0.42$ \\
\hline $\begin{array}{l}\text { service to } \\
\text { others }\end{array}$ & $3.95 \pm 0.44$ & $3.52 \pm 0.39$ & $3.71 \pm 0.33$ \\
\hline
\end{tabular}

\subsection{Correlation among Nurse Image, Ego-resilience, and Nursing Professional Values of} Subjects

The correlations among Nurse image, Ego-resilience, and Nursing professional values of the subjects were analyzed. Nursing professional values were shown to have significant static correlations with Nurse image $(\mathrm{r}=.640, p<.001)$ and Ego-resilience $(\mathrm{r}=.276, p=.012)$, and Nurse image and Ego-resilience had a significant static correlation ( $\mathrm{r}=.264, p<.001)$ (see [Table 4]).

[Table 4] Correlation among Nurse Image, Ego-resilience, and Nursing Professional Values ( $\mathrm{N}=193)$

\begin{tabular}{c|c|c|c}
\hline Variables & Nurse image $r(p)$ & Ego-resilience $\mathrm{r}(p)$ & $\begin{array}{c}\text { Nursing } \\
\text { professional values } \mathrm{r}(p)\end{array}$ \\
\hline \hline Nurse image & 1 & $.264(<.001)$ & $.640(<.001)$ \\
Ego-resilience & & 1 & $.276(.012)$ \\
Nursing professional values & & & 1 \\
\hline
\end{tabular}

\subsection{Factors Influencing Nursing professional values of subjects}

To determine factors influencing Nursing professional values of the subjects, this study performed a regression analysis with Nurse image, and Ego-resilience, which showed significant differences. The tolerance limit was .765 to .967 , which was less than 1.0 , and the variance inflation factor (VIF) was 1.035 to 1.306 , which was less than 10, indicating there no problems of multicollinearity. As for the independence of residuals, the Durbin-Watson value was 1.902, which was close to 2, indicating there was no problem with autocorrelation. The regression model was statistically significant $(\mathrm{F}=171.320, p<.001)$, and the explanatory power of the model was $56.7 \%$. The factors influencing the Nursing professional values of nursing students were Nurse image $(\beta=.636, \mathrm{t}=16.521, p<.001)$ and Ego-resilience $(\beta=.075, \mathrm{t}=2.329, p=.032)$ (see [Table 5]). 
[Table 5] Factors Influencing Nursing Professional Values of Subjects

$(\mathrm{N}=193)$

\begin{tabular}{l|c|c|c|l|l|l|l|l}
\hline Variables & $\mathrm{B}$ & $\mathrm{SE}$ & $\beta$ & $\mathrm{t}(p)$ & $\begin{array}{c}\text { Durbin- } \\
\text { Watson }\end{array}$ & $\mathrm{R}^{2}$ & Adj $\mathrm{R}^{2}$ & $\mathrm{~F}(p)$ \\
\hline \hline Constant & 16.152 & $\begin{array}{c}3.642 \\
.020\end{array}$ & .636 & $\begin{array}{l}3.523(<.001) \\
16.521(<.001)\end{array}$ & 1.673 & .572 & .567 & $\begin{array}{l}171.320 \\
(<.001)\end{array}$ \\
Nurse image & .320 & .025 & .075 & $2.329(.032)$ & & & \\
Ego-resilience & .120 & .035 & \\
\hline
\end{tabular}

\section{Discussion}

This study was attempted to provide a basic data for preparing intervention measures in order to establish Nursing professional values of nursing students by determining the relationships between Nurse image, Ego-resilience, and Nursing professional values of nursing students and identifying the factors influencing Nursing professional values.

In this study, the Nurse image based on the general characteristics showed significant differences in personality and thinking for nursing professionalism. The scores of Nurse image were higher in the students with very optimistic personality than those with optimistic or pessimistic personality and in the students who were optimistic about nursing professionalism than those who were pessimistic. Jeon et al.[26] reported that there were significant differences in sex, personality, and thinking for nursing professionalism and the scores of Nurse image were higher in optimistic students than pessimistic ones. Despite the difficulty of direct comparison due to the differences in general characteristics, the results were partially consistent with those of this study. Ha et al.[27] found that Nurse image was significantly different based on grade and religion; the scores were somewhat higher in sophomores than in freshmen and juniors, and in religious students than in non-religious ones. Lee and Park[15] showed that the scores were highest in sophomores, and higher in religious students than in non-religious ones. The subjects of this study were juniors and seniors, and the scores were slightly higher in the juniors than in the seniors. The results of this study are difficult to compare directly with the precedent studies due to the difference in the subject. However, the sophomores, who had not yet undergone clinical practice, showed higher Nurse image scores than the juniors and seniors in the precedent studies, suggesting that clinical practice may not have a positive influence on Nurse image. Considering the results of the precedent studies that students with high satisfaction with their major and clinical practice have high Nurse image[28], it may be necessary to establish an educational program to enhance the satisfaction of nursing students in theoretical and practical courses. Despite the difficulty in direct comparison due to the difference in research tools, the average point of Nurse image of nursing students in this study was 3.87, showing a higher figure than that of Lee and Park[15] (3.60), Jeon et al.[26] (3.84), 
and Seong et al.[29] (3.85), and slightly lower than Lee[28] and Lim and Jo[16] (4.01).

Ego-resilience was found to be significantly different according to age, sex, grade, personality, thinking for nursing professionalism, and application motivation. The scores of Ego-resilience were highest in the age group of 25-29 years and higher in male, indicating that the results of this study were consistent with those of the precedent studies[18][23]. In terms of the grade, juniors showed higher Ego-resilience than seniors. Although this study did not cover all grades and accordingly the results were insufficient to be compared to those of the precedent studies, which reported that freshmen had higher Ego-resilience than sophomores, juniors, and seniors[30]. So the results of this study were partially consistent with those of the precedent studies in that the Ego-resilience scores were higher in lower-graders than in higher-graders. In terms of personality, "Very optimistic" showed higher Ego-resilience than "Optimistic" and "Pessimistic," indicating that the results of this study were consistent with those of a precedent study[17] reporting that extroverted students had higher Ego-resilience than introverted ones. In terms of application motivation, the subjects selecting "Aptitude" had the highest Ego-resilience. Despite the slight difference in the range of general characteristics, this study result may be in the same context as that of Kang et al.[18], in which students choosing "I was attracted to nursing as a career" showed the highest Ego-resilience. Despite the difficulty in direct comparison due to the difference in research tools, the average point of Ego-resilience in this study was 3.37, showing a higher figure than that of Hwang and Shin[19] (2.95) and Shin and lower than Lee and Kim[17] (3.51), and Kang et al.[18] (3.41).

Nursing professional values exhibited significant differences based on grade, personality, and thinking for nursing professionalism. The juniors showed slightly higher scores than the seniors in terms of Nursing professional values. As reported by the precedent studies[8][19][30], this result may be because the nursing students had high Nursing professional values before clinical practice but then they were influenced by negative factors when they witnessed and experienced duty performance and roles of nurses in hospitals during the practice. The increase in the enrollment quota of nursing colleges is not an appropriate solution; the reduction may suggest how desperate it is to secure professionalism of nurses and to change awareness of nurses by giving a better treatment to nurses and improving the environment of clinical practice. Some precedent studies reported that students who showed high satisfaction with clinical practice had high Nursing professional values[18][30]. In terms of personality, "Very optimistic" students had high Nursing professional values. This may be in a similar context with the result of a precedent study[17], in which very extroverted students had high Nursing professional values. In terms of thinking for Nursing professionalism, the students selecting 
"Optimistic" showed high scores of Nursing professional values. The average point of the Nursing professional values was 3.68, which is lower than Jung et al.[31] (3.82), a study that adopted the same tool with this study. Despite the difficulty in direct comparison due to the difference in research tools, the average of this study (3.68) was higher than Cho and Kim[30] (3.59) but lower than Gu and Lee[8] (3.76), Seong, Yeom, and Do[29] (3.83), Lee and Kim[17] (3.84), Kang et al.[18] (3.87), and Lim and Jo[16] (4.00). The lower scores in Nursing professional values may be caused by the negative nurse image formed during clinical practice, a practice performed by juniors and seniors who are the subjects of this study.

In the correlations between Nurse image, Ego-resilience, and Nursing professional values, Nurse image and Ego-resilience were statically correlated with Nursing professional values, indicating that the higher Nurse image and Ego-resilience, the higher Nursing professional values. These results were consistent with those of the precedent studies reporting that Nursing professional values were higher as Nurse image was higher[8][16][29][30] or that students with high Ego-resilience had high Nursing professional values[17][18]. These results may suggest the necessity of preparing intervention plans to improve Nurse image and Ego-resilience of nursing students in nursing curricula.

The analysis result of factors influencing Nursing professional values showed that Nurse image and Ego-resilience served as influence factors, with $56.7 \%$ of explanatory power. This result was consistent with that of the precedent studies reporting that Nurse image[16][30] and Ego-resilience[17][18] were influence factors of Nursing professional values.

Based on the results of this study, nursing students may develop their interest in nursing by observing the attitudes of nurses and skilled nurses who treat them during clinical practice. Direct experiences through practice in hospitals affect Nursing professional values, theoretical education on nursing as a major and training of clinical practice should be improved continuously in a way that role models in clinical practice can provide nursing students with positive influence[15].

In this context, nursing education institutions should prepare improvements in nursing education in order to promote Nursing professional values in curricula and extra-curricular programs, and nursing communities should pay attention to and make efforts to provide nursing students with high-quality sites of clinical practice to positively influence the students in establishing Nurse image and Nursing professional values. 


\section{Conclusion}

This study is a descriptive research to provide a basic data for preparing effective educational interventions in order to establish Nursing professional values of nursing students by determining the correlations between Nurse image, Ego-resilience, and Nursing professional values of nursing students and identifying the factors influencing Nursing professional values.

According to the results, Nurse image based on general characteristics showed significant differences in personality and thinking for nursing professionalism, while Ego-resilience showed significant differences in age, sex, grade, personality, thinking for nursing professionalism, and application motivation. Nursing professional values had significant differences in grade, personality, and thinking for nursing professionalism.

As for the correlations between Nurse image, Ego-resilience, and Nursing professional values, Nursing professional values had significant static correlations with Nurse image and Ego-resilience, and there was a significant static correlation between Nurse image and Ego-resilience. When factors influencing Nursing professional values were analyzed, Nurse image and Ego-resilience had significant influences on the Nursing professional values, model's explanatory power was $56.7 \%$.

In the light of these findings and preceding studies, the Nursing professional values of nursing students are related to Nurse image and Ego-resilience. Therefore, it is believed that the Nursing professional values of nursing students can be enhanced if theoretical curricula and extra-curricular programs to improve Nurse image and Ego-resilience are developed and applied appropriately.

\section{Acknowledgement}

This study was supported by research funds from Mokpo Catholic University, 2020.

\section{References}

[1] The Ministry of Health and Welfare, Measures to improve working environment and treatment of nurses, https://www.mohw.go.kr/react/al/sal0301vw.jsp?PAR_MENU_ID=04\&MENU_ID=0403\&page=1\&CONT_SEQ=3 44262, Mar 21 (2018)

[2] S. S. Han, M. H. Kim, E. K. Yun, Factors affecting nursing professionalism, The Journal of Korean Academic 
Society of Nursing Education, (2008), Vol.14, No.1, pp.73-79.

[3] K. Hallin, E. Danielson, Registered nurses' perceptions of their work and professional development, Journal of Advanced Nursing, (2008), Vol.61, No.1, pp.62-70.

[4] Y. R. Kim, A study on the change of the views on nursing profession of the nursing college students, The Korean Journal of Fundamentals of Nursing, (1995), Vol.2, No.2, pp.169-181.

[5] K. O. Lee, H. J. Chae, The relationships between emotional intelligence and college adjustment and nursing professionalism in nursing students, Journal of Digital Convergence, (2016), Vol.14, No.10, pp.355-365.

[6] Y. M. Kwon, E. J. Yeun, A correlation study on nursing professional values, department satisfaction, sociality, self-esteem among nursing students, The journal of Korean Nursing Administration Academic Society, (2007), Vol.13, No.3, pp.285-292.

[7] J. Choi, N. S. Ha, The Relationship among Image of Nurses, Self Esteem and Professional Socialization in Nursing Students, The Journal of Korean Nursing Administration Academic Society, (2009), Vol.15, No.1, pp.54-63.

[8] H. J. Gu, O. S. Lee, The Correlation between nurse's image, biomedical ethics and professionalism in nursing students, Journal of Korean Academia-Industrial cooperation Society, (2015), Vol.16, No.11, pp.7356-7366.

[9] Y. S. Ham, H. S. Kim, I. S. Cho, J. Y. Lim, Affecting factors of nursing professionalism perceived by senior nursing students, The Journal of Korean Academic Society of Nursing Education, (2011), Vol.17, No.3, pp.536-544.

[10] M. S. Koh, T. S. Kim, S. Mim, J. S. Park, Y. H. Yom, S. H. Yoon, M. A. Lee, M, Chong, E. Y. Hong, Introduction to nursing, Korea Seoul: Soomoonsa, (2011)

[11] J. Block, A. M. Kremen, IQ and ego-resiliency: conceptual and empirical connections and separateness, Journal of personality and social psychology, (1996), Vol.70, No.2, pp.349-361.

[12] E. J. Ji, M. R. Bang, H. J. Jeon, Ego Resilience, Communication Ability and Problem-Solving Ability in Nursing Students, The Korean Academic Society of Nursing Education, (2013), Vol.19, No.4, pp.571-579.

[13] J. A. Cho, J. S. Kim, Factors affecting nursing college students' satisfaction with their department, Journal of the Korea Academia-Industrial Cooperation Society, (2016), Vol.17, No.4, pp.587-595.

[14] H. J. Park, Correlations among nursing professionalism, critical thinking disposition and self-leadership in nursing students, The Journal of Korean Academic Society of Nursing Education, (2015), Vol.21, No.2, pp.227-236.

[15] E. S. Lee, M. S. Park, A Study of Convergence Relationships among Nurses' Image, Professional Self-Concept and Nursing Professionalism in Nursing Students, Journal of Digital Convergence, (2017), Vol.15, No.1, pp.311-320.

[16] K. M. Lim, E. J. Jo, Influence of Satisfaction with Clinical practice and Image of nurses on Nursing professionalism of Nursing students, Journal of the Korea Academia-Industrial cooperation Society, (2016), Vol.17, No.4 pp.556-566.

[17] H. N. Lee, J. H. Kim, The influencing factors of Ego-Resilience and Empowerment on Nursing 
Professionalism among Nursing Students, Journal of the Korea Academia-Industrial cooperation Society, (2016), Vol.17, No.8, pp.292-300.

[18] H. S. Kang, Y. S. Kang, Y. M. Kim, The Relationship between Critical Thinking, Ego resilience and Nursing Professionalism among Senior Nursing Students, Asia-pacific Journal of Multimedia Services Convergent with Art, Humanities, and Sociology, (2016), Vol.6, No.4, pp.219-229.

[19] E. H. Hwang, S. J. Shin, Factors Impacting Nursing Professionalism among Nursing Students, Journal of Korean Public Health Nursing, (2017), Vol.31, No.1, pp.97-108.

[20] H. J. Kim, A Study on Image of the Nurse, Chonbuk University, Unpublished Master's Thesis, (2000)

[21] D. S. Lee, A Study Human perceived Nurse's image, Hanyang University, Unpublished Master's Thesis, (1995)

[22] I. S. Yang, A study on the Image of Nurses and Determinants the Image, The Journal of Korean Nursing Administration Academic Society, (1998), Vol.4, No.2, pp.289-305.

[23] I. J. Song, A Comparative Study of the Opinon on the Image of Nursing Among the Doctors, Nurses, other Hospital Personnel and the General Public, The Korean Nurse, (1993), Vol.32, No.2, pp.51-62.

[24] S. K, Yoo, H. W. Shim, Psychological Protective Factors in Resilient Adolescents in Korea, Korean Journal of Education Psychology, (2002), Vol.16, No.4, pp.189-206.

[25] E. J. Yeun, Y. M. Kwon, O. H. Ahn, Development of a Nursing Professional Value Scale, Journal Korean of Academy of Nursing, (2005), Vol.35, No.6, pp.1091-1100.

[26] E. J. Jeon, Y. M. Noh, S. M. Ryou, S. Y. Oh, K. S. Park, Perceived image of nurses on major and non-major students, Journal of the Korea institute of oriental medical informatics, (2005), Vol.21, No.2, pp.1-9.

[27] Y. J. Ha, S. Min, E. A. Kim, The Moderating Effect of Self-Regulatory Efficacy in the Relationship between Self-image and Perception of the Nurse's Image of Nursing Students, The Journal of Korean Academic Society of Nursing Education, (2013), Vol.19 No.3, pp.405-412.

[28] J. Y. Lee, A Study on Nurse Image, Professional Self-concept and Organizational Commitment of Nursing Students, Journal of the Korea Academia-Industrial cooperation Society, (2017), Vol.18, No.3, pp.284-295.

[29] J. A, Seong, E. Y. Yeom and Y. S. Do, Image of Nurses and Nursing Professional Values Perceived by Nursing Students, The Journal of the Korea Contents Association, (2014), Vol.14, No.11, pp.798-809.

[30] H. H. Cho, N. H. Kim, Relationships Among Nursing Professionalism, Nurse Image, and Core Elements of Nursing Professionalism that Nursing Students Perceive, The Journal of Korean Academic Society of Nursing Education, (2014), Vol.20, No.4, pp.548-557.

[31] K. T. Jung, Y. J. Ha, N. Y. Oh, S. Y. Kim, M. J. Kwon, N. H. Lee, Y. R. Lee, K. H. Yang, Factors Affecting Satisfaction with Clinical Practice and Nursing Professional Values in Nursing Students, The Journal of Korean Nursing Research, (2018), Vol.2, No.1, pp.23-36. 